\title{
VLBA Observations of Astrometric Reference Sources in the Galactic Center
}

\author{
Geoffrey C. Bower ${ }^{1}$, Donald C. Backer ${ }^{2}$ \& Richard A. Sramek ${ }^{1}$
}

\begin{abstract}
We report here on multi-frequency VLBA observations of three extragalactic sources within 1 degree of the Galactic Center. These sources have been used as astrometric reference sources for VLA and VLBA determinations of the proper motion of Sagittarius A*, the compact nonthermal radio source in the Galactic Center. Each source has a main component with a brightness temperature in excess of $10^{7.5} \mathrm{~K}$, confirming that the sources are active galactic nuclei. The sources have simple structure that can be characterized by one or two Gaussian components. The frequency dependence of the structure indicates that the positions of Sgr A* determined by the VLA astrometry of Backer \& Sramek (1999) at 4.8 and $8.4 \mathrm{GHz}$ should have an offset of $\sim 2$ mas. This offset is in the same direction as the 5 mas shift measured by Backer \& Sramek (1999). The structure is unlikely to bias the $43 \mathrm{GHz}$ VLBA results of Reid et al. (1999). Motions of components in the calibrator sources could lead to errors in the proper motion of Sgr $\mathrm{A}^{*}$ on the order of a few $\mathrm{km} \mathrm{s}^{-1}$.

All three sources show frequency dependent structure consistent with scattering which is significantly stronger than that of the Galactic scattering model of Taylor \& Cordes (1993) but significantly weaker than that of the hyperstrong Galactic Center scattering. Combined with other observations, this suggests the existence of a new component of Galactic scattering located several kpc from the Galactic Center.
\end{abstract}

Subject headings: Galaxy: center — galaxies: active — scattering

\section{Introduction}

The compact radio source in the Galactic Center, Sagittarius $\mathrm{A}^{*}$, is the best and closest candidate for a massive black hole in the center of a galaxy (Maoz 1998). The source Sgr $A^{*}$ is positionally coincident with a $\sim 2.6 \times 10^{6} M_{\odot}$ dark mass (Genzel et al. 1997; Ghez et al. 1998, 2000). Very long baseline interferometry (VLBI) has shown that this source has a size scale less than $1 \mathrm{AU}$ and a brightness temperature in excess of $10^{9} \mathrm{~K}$ (Rogers et al. 1994; Bower \& Backer 1998; Lo et al. 1998; Krichbaum et al. 1998). For these reasons, it is inferred that $\mathrm{Sgr} \mathrm{A*}$ is a mas-

\footnotetext{
${ }^{1}$ National Radio Astronomy Observatory, P.O. Box O, 1003 Lopezville, Socorro, NM 87801; gbower@nrao.edu,rsramek@nrao.edu

${ }^{2}$ Astronomy Department \& Radio Astronomy Laboratory, University of California, Berkeley, CA 94720; dbacker@astro.berkeley.edu
}

sive black hole surrounded by a synchrotron or cyclo-synchrotron emission region powered by accretion (Melia 1994; Narayan et al. 1998; Falcke et al. 1993; Mahadevan 1998). Nevertheless, significant details regarding the emission region are unknown. In particular, we don't know whether the emission originates in an inflow or an outflow and whether the emission region is optically thin or thick. These may have observational consequences. In particular, some outflow models require frequency-dependent positions while most inflow models give frequency-independent positions.

Long-term astrometric studies indicate that Sgr $A^{*}$ shows a proper motion of $\sim 6$ mas $\mathrm{y}^{-1}$, which is consistent with the Sun's rotation around the Galactic Center and no motion of Sgr A* with respect to the Galactic Center. These results strongly confirm the hypothesis that Sgr A* is a 
massive black hole in the Galactic Center. The Very Large Array (VLA) experiment of Backer \& Sramek (1999) determined the position of Sgr A* with respect to three radio sources within 1 degree over $18 \mathrm{y}$ at $4.8 \mathrm{GHz}$ and over $10 \mathrm{y}$ at $8.4 \mathrm{GHz}$. Reid et al. (1999) measured the position of Sgr A* with respect to two of these same sources over $2 y$ with the Very Long Baseline Array (VLBA) at $43 \mathrm{GHz}$. Both experiments assume that the reference sources are extragalactic and have frequencyindependent, time-invariant positions. In one of their highest quality epochs, Backer \& Sramek (1999) also found a 5 mas offset between the 4.8 and $8.4 \mathrm{GHz}$ positions of Sgr A*. A portion or all of this could be the result of source structure as we will discuss below. However, since the effect is $\sim 0.01$ times the beam size of the VLA and is $\sim 0.1$ times the scattering size, systematic errors and refraction could contribute to the offset.

Interstellar scattering of the radiation along the line of sight broadens the image of Sgr $\mathrm{A}^{*}$ and other Galactic Center sources at radio through millimeter wavelengths (e.g., Lo et al. 1998; Frail et al. 1994; Lazio \& Cordes 1998a). The scattering medium has been modeled as a plasma at a distance of $\sim 150 \mathrm{pc}$ from the Galactic Center with a radial extent in Galactic latitude and longitude on the order of $0.5^{\circ}$, using constraints from the scattering sizes of sources physically located in the Galactic Center, the emission measure of the plasma and surveys for background extragalactic sources. The size of Sgr $\mathrm{A}^{*}$ is $\sim 1^{\prime \prime}$ at $1 \mathrm{GHz}$. An extragalactic source behind the scattering screen will have a size $\sim 100^{\prime \prime}$ since the scattering of Sgr $A^{*}$ is very inefficient due to its proximity to the scattering region. The scattering medium, which is often referred to as the hyperstrong scattering medium, requires a turbulent electron density several orders of magnitude greater than that predicted by the best model for galactic electron content (Taylor \& Cordes 1993). The model predicts that extragalactic sources in the Galactic Center will have a size of only $\sim 0.1^{\prime \prime}$ at $1 \mathrm{GHz}$. The model is known to be inaccurate in the Galactic Center because it excludes scattered sources to avoid confusion with the hyperstrong scattering region and because it relies on pulsar data which lack sensitivity beyond $\sim 4 \mathrm{kpc}$.

We present here VLBA observations at 2.3 $\mathrm{GHz}, 5.0 \mathrm{GHz}$ and $8.4 \mathrm{GHz}$ of the three astromet- ric reference sources of Backer \& Sramek (1999). In Section 2 we summarize the observations and results. In Section 3 we show that the sources are very likely to be extragalactic. In Section 4 we discuss the impact of these observations on the VLA and VLBA astrometric results. In Section 5 we show that the sources are scattered and discuss the medium responsible for the scattering. We give our conclusions in Section 6 .

\section{VLBA and VLA Observations and Re- sults}

Observations were made with the VLBA from 2300 UT 29 August 1999 to 0600 UT 30 August 1999. A single VLA antenna was also included to increase sensitivity to large-scale structure. Sky frequencies of 2.3, 5.0 and 8.4 $\mathrm{GHz}$ were observed with total sample rates of $128 \mathrm{Mbits} \mathrm{s}^{-1}$. The VLA did not observe at 2.3 GHz. We cycled frequently through the sources W56 (B1742-283=J1745-2820), W109 (B1745291=J1748-2907), GC 441 (B1737-294=J17402929) and Sgr A* at the three separate frequencies. See Table 1 for source positions. Hourly observations of NRAO 530 (J1733-1302) were also made at each frequency. The data were correlated in Socorro, New Mexico.

Analysis was performed with the AIPS package. A priori amplitude calibration was applied using measured system temperatures and standard gain curves. High SNR fringes were detected on all baselines for NRAO 530. Fringes were also found on short baselines for the four other sources at 5.0 and $8.4 \mathrm{GHz}$. At $2.3 \mathrm{GHz}$ fringes were detected consistently for GC 441 only. No fringes were detected for Sgr A* and W56 at $2.3 \mathrm{GHz}$. An attempt was made to reference the phase of the different sources. This failed due to ionospheric phase fluctuations and the small number of baselines. Instead, the visibility data were phase selfcalibrated before imaging. Thus, absolute position information is not recovered in these observations. We present the results of fitting Gaussian models to the visibility data in Table 2 . Zero baseline fluxes determined by VLA observations on 10 April 1998 (Table 3) indicate that $90 \pm 10 \%$ of the flux is recovered in the VLBA images at 5.0 and $8.4 \mathrm{GHz}$ for all sources. Monitoring of the calibrator fluxes over the past $20 \mathrm{y}$ shows that these 
sources are slowly variable (Bower et al. 2001). Images of GC 441 at 5.0 and $8.4 \mathrm{GHz}$ are shown in Figure 1.

We also present in Table 3 the results of linear polarimetric observations at 4.8 and $8.4 \mathrm{GHz}$ with the VLA of these sources and the source J1751253 on 10 April 1998. The $4.8 \mathrm{GHz}$ results were previously presented in Bower et al. (1999). The polarization position angles were calibrated with observations of 3C 286. We calculate a rotation measure (RM) for each source. The error in position angle is dominated by calibration and is on the order of a few degrees. The error in RM is on the order of $10 \mathrm{rad} \mathrm{m}^{-2}$. The sources are compact in these A array observations, implying sizes less than 300 mas. There is some evidence in the visibilities for diffuse structure around W109 but it is not clear if this is physically associated with the source.

\section{The AGN Nature of the Reference Sources}

The brightness temperature for each source component is listed in Table 2. For the brightest components in each source, $T_{b} \gtrsim 10^{7.5} \mathrm{~K}$. Supernovae (e.g., Marcaide et al. 1997) and galactic stellar-mass black hole sources (e.g., Hjellming \& Rupen 1995) are the only galactic sources known to have brightness temperatures this large. However, each of these display light curves inconsistent with the roughly constant flux of the reference sources over the past 20y (Bower et al. 2001; Weiler et al. 1998; Waltman et al. 1999).

The sources GC 441 and W56 resemble extragalactic jet sources with a flat spectrum core (component A) and a steep spectrum jet (component B), while W109 resembles an unresolved flat spectrum radio core. The spectral index for GC 441B is $\alpha=-1.2 \pm 0.4\left(S_{\nu} \propto \nu^{\alpha}\right)$. We can only find an upper limit on $\alpha$ for W56B, which was not detected at $8.4 \mathrm{GHz}$. The rms in the map at the location of $\mathrm{W} 56 \mathrm{~B}$ is $0.140 \mathrm{mJy}^{\text {beam }}{ }^{-1}$. The lack of a detection of W56B at the $5 \sigma$ level implies $\alpha<-2.4 \pm 0.7$, which indicates that this component is almost certainly heavily resolved at 8.4 $\mathrm{GHz}$. The fluxes of the secondary components were too low to obtain reliable fits for their sizes in either the image or the visibility planes.

Given the absence of resemblance to known galactic sources and the clear resemblance to extragalactic jet sources, we conclude that the sources are extragalactic. This strongly supports the assumptions that underly the astrometric conclusions of Backer \& Sramek (1999) and Reid et al. (1999).

\section{The Differential Astrometric Position of Sagittarius A*}

The sources W56 and GC 441 have asymmetric structure that is frequency dependent. GC 441 is extended to the Northwest and W56 is extended to the Southwest. Both sources have greater asymmetry at $5.0 \mathrm{GHz}$ than at $8.4 \mathrm{GHz}$. The effect of these asymmetries is that the astrometrically measured position of Sgr A* relative to these reference sources at $5.0 \mathrm{GHz}$ will be to the East of that at $8.4 \mathrm{GHz}$. While our VLBA results do not have absolute or relative astrometry between the sources, we can explore how the reference source centroids, which are what the VLA observations measure, vary between frequencies. Consequently we can estimate systematic differences in the VLA differential astrometry of Sgr A*.

We can estimate the frequency-dependent positions by computing the centroids of the images. In Table 4 we report the difference in the centroids of the emission with the peak of the emission at each of the two frequencies $\left(\Delta \alpha_{C}\right.$ and $\Delta \delta_{C}$ at $5.0 \mathrm{GHz}$ and $\Delta \alpha_{X}$ and $\Delta \delta_{X}$ at $\left.8.4 \mathrm{GHz}\right)$. We then give the difference in these terms, $\Delta \alpha_{C X}=\Delta \alpha_{C}-\Delta \alpha_{X}$ and $\Delta \delta_{C X}=\Delta \delta_{C}-\Delta \delta_{X}$, as estimates of the difference in the frequency-dependent positions. The results for $\mathrm{Sgr} \mathrm{A}^{*}$ and W109 serve as estimates of the error due to noise in the map. However, as we discuss below, the total error is probably dominated by resolution and opacity effects. Combining the frequency-dependent positions with the weighting applied by Backer \& Sramek (1999) we find that the measured position of Sgr $\mathrm{A}^{*}$ at 4.8 $\mathrm{GHz}$ will be $2.0 \pm 0.1$ mas to the East and $0.6 \pm 0.4$ mas to the North of the position at $8.4 \mathrm{GHz}$. Backer \& Sramek (1999) found in a single epoch that the $4.8 \mathrm{GHz}$ position was $\sim 5$ mas to the East of the $8.4 \mathrm{GHz}$. The agreement in sign and order of magnitude between these results suggests that we are accounting for the dominant effect.

The discrepancy in magnitude can be explained by the difficulties of estimating positions with the 
VLA to mas-accuracy, by missing zero-baseline flux in the VLBA results, and by offsets in the peak of emission at the different frequencies. The missing zero-baseline flux is almost certainly distributed along the principal axes of the two component sources. For example, we see a suggestion in the $5.0 \mathrm{GHz}$ image of GC 441 of an additional weak component at a distance of 100 mas along the axis of the jet. Since jet sources are likely to be more extended at lower frequencies, any additional flux will bias the results to a larger angle. Offsets in emission peaks are also likely to play a role at sub-mas scales since the source cores are optically thick. Rioja et al. (1997) have measured an offset in the peak of emission at $13 \mathrm{~cm}$ and $3.6 \mathrm{~cm}$ of 0.7 mas of the extragalactic radio source $1038+52 \mathrm{~A}$, for example.

The differential position of Sgr $\mathrm{A}^{*}$ determined at $4.8 \mathrm{GHz}$ will differ from that at $43 \mathrm{GHz}$, as well. This difference can be computed assuming that all sources have single components at $43 \mathrm{GHz}$. The expected difference then between the Backer \& Sramek (1999) position and the Reid et al. (1999) position is 2.3 mas to the East and 0.6 mas to the North. This is less than the error in the measured absolute position of $\mathrm{Sgr} \mathrm{A}^{*}$, which is $\sim 5$ mas.

Structural variability in the sources could impact the frequency-dependent position and measured proper motion of Sgr A*. Assuming a proper motion of 1 mas $^{-1}$ (Vermeulen \& Cohen 1994) for the GC 441B and W56B components, we can estimate the evolution of the frequency-dependent position and an offset in the proper motion of Sgr A*. Using a weighted average of source positions, we find a potential bias in the measured proper motion of $\mathrm{Sgr} \mathrm{A}^{*}$ at $5.0 \mathrm{GHz}$ of 0.1 mas $\mathrm{y}^{-1}$ over the past $20 \mathrm{y}$. The proper motion of Sgr $\mathrm{A}^{*}$ would become more negative in right ascension and more positive in declination, in roughly equal parts. This brings the measured proper motions of Backer \& Sramek (1999) and Reid et al. (1999) slightly closer together. The differences, however, are still greater than the $1 \sigma$ errors in the right ascension proper motion. For both sources, the flux evolution of the A and B components could have as substantial an impact on the centroids as their proper motion does.

Motion of currently undetectable components closer to the cores of the sources could have a much more significant impact on the proper mo- tion of Sgr A*. This is true for both the 4.8 and 43 $\mathrm{GHz}$ results. Additionally, many sources at higher frequencies are known to have variable structure on timescales of weeks to months (e.g., Marscher et al. 1999; Bower et al. 1997). Predicting the direction of these proper motion offsets is not possible with these observations, either. First, we have observed no preferred angle for the source W109. Second, many sources show bent or misaligned jets between milliarcsecond and arcsecond scales, or have misalignments between different frequencies (Pearson \& Readhead 1988). This implies that proper motion measurements with accuracies better than 0.1 mas $\mathrm{y}^{-1}$ must be accompanied by high resolution imaging of the astrometric reference sources.

\section{Scattering of Sources in the Galactic Center Region}

\subsection{Angular Broadening}

In the Appendix of his review Rickett has formulated the dependence of apparent source visibility of point sources on the turbulent properties of the intervening medium (Rickett 1990). Angular scattering in the intervening medium leads to apparent normalized visibility for a point source of the form

$$
\Gamma(\bar{b})=e^{-\frac{1}{2} D(\bar{b})},
$$

where $D(\bar{b})=A_{\nu} b_{x}^{\alpha}+B_{\nu} b_{y}^{\alpha}$ is the phase structure function of the scattering region, and $\bar{b}=\left(b_{x}, b_{y}\right)$ is the vector projected baseline length. This formulation allows for the general case of asymmetric turbulence with unequal amplitudes along orthogonal, principal axes $A_{\nu} \neq B_{\nu}$; see Rickett (1990) for definition of these amplitudes. The structure function also depends quadratically on the inverse radio frequency owing to the cold plasma dispersion law $A_{\nu}, B_{\nu} \propto \nu^{-2}$. For the extreme scattering case which is present in the Galactic Center and with projected baselines less than the inner scale of the turbulence, we expect $\alpha$ equals exactly 2. Therefore, the model predicts Gaussian components whose size depends quadratically on inverse frequency. The observed frequency dependence of the images then provides a check on $\alpha=2$.

The three extragalactic sources and Sgr A* show clear evidence for scattering in their size as a function of frequency (Figure 2). The sizes for 
Sgr A* are consistent with those measured previously at these and other frequencies (e.g., Bower $\&$ Backer 1998; Lo et al. 1998). For $\sigma_{m a j} \propto \nu^{-\alpha}$, $\alpha_{S g r A^{*}}=1.90 \pm 0.04, \alpha_{G C 441}=1.78 \pm 0.34$, $\alpha_{W 56}=1.98 \pm 0.14$, and $\alpha_{W 109}=1.96 \pm 0.13$. These are reasonably consistent with $\alpha=2$ or slightly less. They are marginally consistent with the Kolmogorov case for baselines greater than the inner scale, $\alpha=1.67$. The constant axial ratios also imply that the minor axes of these sources follow $\alpha=2$. In addition, the position angles $\phi$ are consistent with no change with wavelength. Results for GC 441B and W56B are roughly consistent with the scattering but are difficult to characterize quantitatively due to low SNR.

Additionally, fits to the visibility data at each frequency with $\alpha$ unconstrained consistently found $\alpha=2.0$ for the main components of each source. This confirms that the source sizes found are not confused by extended structure and that the inner scale of the Kolmogorov turbulence distribution is greater than the longest effective baseline on which detections were made (e.g., Wilkinson et al. 1994). This is on the order of $20 M \lambda$ at $8.4 \mathrm{GHz}$, which is $700 \mathrm{~km}$. In the case of Sgr A*, the effective baseline is the baseline length scaled by the ratio $f$ of the distance of the source to the scattering screen to the distance of the source to the observer. For Sgr $A^{*}$, this ratio is on the order of 0.01 , implying an inner scale greater than $10 \mathrm{~km}$.

\subsection{The Intermediate Strength Scattering Region}

The scattering sizes of the three extragalactic sources are two orders of magnitude less than what is expected for an extragalactic source behind the hyperstrong scattering region of the Galactic Center. At $5 \mathrm{GHz}$, this size is $\sim 4^{\prime \prime}$ (Lazio \& Cordes 1998a). Thus, these sources either delineate the outer limits of the hyperstrong Galactic Center scattering region or are viewed through holes in the hyperstrong scattering region. We favor the former explanation. Each of these sources was at the center of a $30^{\prime}$-diameter field surveyed at $20 \mathrm{~cm}$ by Lazio \& Cordes (1998b). The number of sources found in each field $(>12)$ was significantly in excess of the number of extragalactic sources expected $(\sim 5)$ in the absence of the hyperstrong scattering region, indicating that the entire fields, or substantial parts, are outside of the hyperstrong scattering region. Fields closer to Sgr $A^{*}$ show significantly fewer or no sources. In order for holes in the scattering medium to explain the weaker scattering, the scale of patchiness must be significantly less than the field size, $30^{\prime}$, and the hyperstrong scattering region must also cover a small fraction of the fields. Lazio et al. (1999) estimate the scale of patchiness at $5^{\prime}$ from the proximity of strongly scattered galactic sources to the extragalactic source 1LC359.872+0.178, which is not scattered by the hyperstrong scattering region. However, the covering factor of the hyperstrong scattering region in the field surrounding this source must be much higher than in our fields, since this source is the only one found in this field. We conclude that the reference sources are outside of the hyperstrong scattering region. They do not uniquely define the extent in latitude and longitude, but they do suggest the region is bounded by $359^{\circ}<l<1^{\circ}$ and $|b|<0.5^{\circ}$.

The scattering sizes of the reference sources are a factor of 1.5 to 6 greater than predicted by the galactic scattering model of Taylor \& Cordes (1993). This is unsurprising given the lack of sensitivity in the Taylor \& Cordes (1993) model to the Galactic Center scattering. These results suggest the existence of a scattering region of intermediate strength between the Taylor \& Cordes (1993) model and the hyperstrong Galactic Center scattering.

We plot in Figure 3 the scattering sizes of our sources along with those of several extragalactic sources and $\mathrm{OH} 1612 \mathrm{MHz}$ maser spots from van Langevelde et al. (1992). For clarity we exclude scattered sources within $0.25^{\circ}$ of $\mathrm{Sgr} \mathrm{A}^{*}$ which are clearly associated with the hyperstrong scattering region (Frail et al. 1994; Yusef-Zadeh et al. 1999). The extent of the region of intermediatestrength scattering is not well constrained. The scattering sizes of our sources are similar to those of some extragalactic sources in the vicinity of the Galactic Center [e.g., J1744-312 $\left(l=357.86^{\circ}, b=\right.$ $\left.-1.00^{\circ}\right), \mathrm{B} 1739-298\left(l=358.92^{\circ}, b=0.07^{\circ}\right)$ (Lazio \& Cordes 1998b) and 1LC 359.872+0.178 (Lazio et al. 1999)].

Other, more weakly scattered sources provide outer bounds to the intermediate strength scattering region. We can limit its extent in Galactic latitude with sources from the VLBA calibrator survey (Peck \& Beasley 1998): J1700-2610 ( $l=$ 
$\left.356.73^{\circ}, b=10.02^{\circ}\right), \mathrm{J} 1713-2658\left(l=357.67^{\circ}, b=\right.$ $\left.7.10^{\circ}\right)$ and $\mathrm{J} 1820-2528\left(l=6.79^{\circ}, b=-4.94^{\circ}\right)$. At $4 \mathrm{~cm}$ all show flat amplitudes on VLBA baselines as long as $250 M \lambda$, indicating compact component sizes less than 1 mas. Together, these provide an upper limit of $5^{\circ}$ in latitude for the intermediatestrength scattering region.

The SM is greater at negative longitudes than at positive longitudes. For negative longitudes, the extent in longitude of the intermediatestrength scattering is $\gtrsim 5^{\circ}$. van Langevelde et al. (1992) found $\mathrm{OH} 1612 \mathrm{MHz}$ masers with sizes of $\sim 350$ mas at negative longitudes as great as $6.7^{\circ}$. Maser sizes for $l>+1.3^{\circ}$ are typically 100 mas, corresponding to 10 mas at $5 \mathrm{GHz}$. This is supported by the lack of intermediate-strength scattering in $\mathrm{J} 1751-253\left(l=3.73^{\circ}, b=0.85^{\circ}\right)$. This extragalactic object is known to be scattered ( R. Hjellming, W. Brisken, private communications) with a size at $1.6 \mathrm{GHz}$ of 40 mas, consistent with the prediction of Taylor \& Cordes (1993).

The similar degree of scattering of the Galactic Center $\mathrm{OH}$ sources and the extragalactic sources implies that the scattering must be distant from the Galactic Center. This is unlike the case of the hyperstrong scattering region which is $\sim 150 \mathrm{pc}$ from the Galactic Center.

The intermediate-strength scattering region in the Galactic Center is stronger than other strong scattering regions in the Galaxy. Enhanced scattering in the Cygnus region covers $50^{\circ}<l<70^{\circ}$ and produces scattering sizes of $\sim 5$ mas at $5 \mathrm{GHz}$ (Fey et al. 1991; Desai \& Fey 2000). OH $1720 \mathrm{MHz}$ masers seen in W28 and W44 may also be scattered with typical sizes of 100 mas, corresponding to a scattering size of $\sim 10$ mas at $5 \mathrm{GHz}$ (Claussen et al. 1999). OH $1612 \mathrm{MHz}$ masers in the W49 region are scattered on the scale of 100 to 200 mas (Desai et al. 1994). Potentially, the Galactic masers are experiencing near-field scattering. In this case, the strength of scattering in these regions may equal or exceed that of the intermediate-strength scattering region.

Only two other individual extragalactic sources exhibit scattering as strong as we see for the Galactic Center reference sources. As mentioned above, B1849+005 has a size on the order of 45 mas at $5 \mathrm{GHz}$ (Fey et al. 1991). Additionally, the source NGC 6334B is very heavily scattered with a size of $3^{\prime \prime}$ at $1.5 \mathrm{GHz}$ (Trotter et al. 1998). The an- gular extent of the scattering region around NGC $6334 \mathrm{~B}$ is unknown. However, $\mathrm{H}_{2} \mathrm{O}$ masers within $2^{\prime}$ are unscattered.

\section{Conclusions}

We have imaged three astrometric reference sources in the Galactic Center. We have four principal conclusions.

1. Based on their morphologies, brightness temperatures and steady fluxes, we conclude that these sources are active galactic nuclei. This supports the basic astrometric conclusions of Backer \& Sramek (1999) and Reid et al. (1999).

2. Two of the three sources are asymmetric. The frequency dependence of this asymmetry accounts for the measured frequency-dependent position of $\operatorname{Sgr} A^{*}$. However, we are unable to quantitatively determine what residual frequencydependence there may be in the position of Sgr A* Thus, we cannot distinguish between optically thick jet models and optically thin accretion models based on these data. A new result requires denser $(u, v)$ coverage on baselines less than 300 $\mathrm{km}$. The proposed A+ array of the expanded VLA will be ideal for making these measurements.

3. Proper motion of components in the reference sources could contribute 0.1 mas $^{-1}$ to the measured proper motion of Sgr A*. This may account for some of the discrepancy between the results of Backer \& Sramek (1999) and Reid et al. (1999).

4. The sources are all scattered. All three are scattered much less than expected from the hyperstrong Galactic Center scattering region. Two of the three, W56 and W109, are scattered much more than expected by the galactic electron distribution model of Taylor \& Cordes (1993). This suggests the existence of an intermediate strength scattering region covering the Galactic Center. Several other known sources are also apparently scattered by this region. We infer that the region is several kpc away from the Galactic Center, that it covers $\gtrsim 5^{\circ}$ in longitude and $<5^{\circ}$ in latitude. This is one of the strongest known scattering regions in the Galaxy. Its covering factor, extent and relationship to the hyperstrong scattering screen can be further probed with observations of masers in the Galactic Center and background extragalactic sources. 
This final result points to a general deficiency in our knowledge of scattering in the Galaxy. The global models for electron distribution were based on scattering observations made before the advent of the VLBA. The VLBA is capable of detecting and imaging a much greater number of sources over a broad frequency range. This would lead to a Galactic electron model of significantly greater accuracy and angular resolution.

The National Radio Astronomy Observatory is a facility of the National Science Foundation operated under cooperative agreement by Associated Universities, Inc. We thank Ketan Desai for assistance with fitting models to the visibilities.

\section{REFERENCES}

Backer, D. C. \& Sramek, R. A. 1999, ApJ, 524, 805

Bower, G. C. \& Backer, D. C. 1998, ApJ, 496, L97

Bower, G. C., Backer, D. C., Wright, M., Forster, J. R., Aller, H. D., \& Aller, M. F. 1997, ApJ, 484, 118

Bower, G. C., Backer, D. C., Zhao, J. H., Goss, M., \& Falcke, H. 1999, ApJ, 521, 582

Bower, G. C., Falcke, H., Sault, R. J., \& Backer, D. C. 2001, ApJ in preparation

Claussen, M. J., Goss, W. M., Frail, D. A., \& Desai, K. 1999, ApJ, 522, 349

Desai, K. \& Fey, A. L. 2000, ApJ submitted

Desai, K. M., Gwinn, C. R., \& Diamond, P. J. 1994, Nature, 372, 754

Falcke, H., Mannheim, K., \& Biermann, P. L. 1993, A\&A, 278, L1

Fey, A. L., Spangler, S. R., \& Cordes, J. M. 1991, ApJ, 372, 132

Frail, D. A., Diamond, P. J., Cordes, J. M., \& van Langevelde, H. J. 1994, ApJ, 427, L43

Genzel, R., Eckart, A., Ott, T., \& Eisenhauer, F. 1997, MNRAS, 291, 219

Ghez, A. M., Klein, B. L., Morris, M., \& Becklin, E. E. 1998, ApJ, 509, 678
Ghez, A. M., Morris, M., Becklin, E. E., Tanner, A., \& Kremenek, T. 2000, Nature, 407, 349

Hjellming, R. M. \& Rupen, M. P. 1995, Nature, 375,464

Krichbaum, T. P., Graham, D. A., Witzel, A., Greve, A., Wink, J. E., Grewing, M., Colomer, F., de Vicente, P., Gomez-Gonzalez, J., Baudry, A., \& Zensus, J. A. 1998, A\&A, 335, L106

Lazio, T. J. W., Anantharamaiah, K. R., Goss, W. M., Kassim, N. E., \& Cordes, J. M. 1999, ApJ, 515, 196

Lazio, T. J. W. \& Cordes, J. M. 1998b, ApJS, 118, 201

-. 1998a, ApJ, 505, 715

Lo, K. Y., Shen, Z. Q., Zhao, J. H., \& Ho, P. T. P. 1998, ApJ, 508, L61

Mahadevan, R. 1998, Nature, 394, 651

Maoz, E. 1998, ApJ, 494, L181

Marcaide, J. M., Alberdi, A., Ros, E., Diamond, P., Shapiro, I. I., Guirado, J. C., Jones, D. L., Mantovani, F., Perez-Torres, M. A., Preston, R. A., Schilizzi, R. T., Sramek, R. A., Trigilio, C., van Dyk, S. D., Weiler, K. W., \& Whitney, A. R. 1997, ApJ, 486, L31

Marscher, A. P., Marchenko, S. G., Gomez, J. L., Cawthorne, T. V., Stevens, J. A., Gear, W. G., Robson, E. I., Lister, M. L., Gabuzda, D. C., Smith, P. S., \& Forster, J. R. 1999, in American Astronomical Society Meeting, Vol. 194, 6203

Melia, F. 1994, ApJ, 426, 577

Narayan, R., Mahadevan, R., Grindlay, J. E., Popham, R. G., \& Gammie, C. 1998, ApJ, 492, 554

Pearson, T. J. \& Readhead, A. C. S. 1988, ApJ, 328,114

Peck, A. B. \& Beasley, A. J. 1998, in ASP Conf. Ser. 144: IAU Colloq. 164: Radio Emission from Galactic and Extragalactic Compact Sources, 155 
Reid, M. J., Readhead, A. C. S., Vermeulen, R. C., \& Treuhaft, R. N. 1999, ApJ, 524, 816

Rickett, B. J. 1990, ARA\&A, 28, 561

Rioja, M. J., Marcaide, J. M., Elosegui, P., \& Shapiro, I. I. 1997, A\&A, 325, 383

Rogers, A. E. E., Doeleman, S., Wright, M. C. H., Bower, G. C., Backer, D. C., Padin, S., Philips, J. A., Emerson, D. T., Greenhill, L., Moran, J. M., \& Kellermann, K. I. 1994, ApJ, 434, L59

Taylor, J. H. \& Cordes, J. M. 1993, ApJ, 411, 674

Trotter, A. S., Moran, J. M., \& Rodriguez, L. F. 1998, ApJ, 493, 666

van Langevelde, H. J., Frail, D. A., Cordes, J. M., \& Diamond, P. J. 1992, ApJ, 396, 686

Vermeulen, R. C. \& Cohen, M. H. 1994, ApJ, 430, 467

Waltman, E. B., Ghigo, F. D., Hjellming, R. M., \& Johnston, K. J. 1999, in American Astronomical Society Meeting, Vol. 194, 4802

Weiler, K. W., van Dyk, S. D., Montes, M. J., Panagia, N., \& Sramek, R. A. 1998, ApJ, 500, 51

Wilkinson, P. N., Narayan, R., \& Spencer, R. E. 1994, MNRAS, 269, 67

Yusef-Zadeh, F., Roberts, D. A., Goss, W. M., Frail, D. A., \& Green, A. J. 1999, ApJ, 512, 230


Fig. 1. - VLBA images of GC 441 at (a) 5.0 and (b) $8.4 \mathrm{GHz}$. The contour levels are -2 , $2,4,8,16,32$ and $64 \%$ of the peak intensity of $20.1 \mathrm{mJy}^{\text {beam }}{ }^{-1}$ at $5.0 \mathrm{GHz}$ and $-1,1,2$, $4,8,16,32$ and $64 \%$ of the peak intensity of $16.9 \mathrm{mJy} \mathrm{beam}^{-1}$ at $8.4 \mathrm{GHz}$. Image rms noise is $170 \mu \mathrm{Jy} \mathrm{beam}^{-1}$ at $5.0 \mathrm{GHz}$ and $80 \mu \mathrm{Jy} \mathrm{beam}^{-1}$ at $8.4 \mathrm{GHz}$. Synthetic beams are shown in the lower left hand corners. 


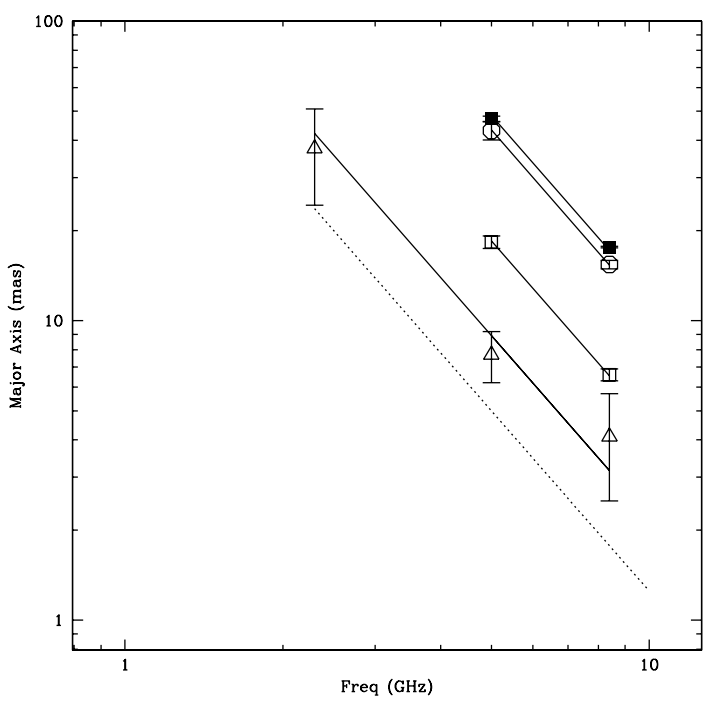

Fig. 2.- The major axis size of the sources plotted as a function of frequency. Open triangles are for GC 441A, open squares are for W109, open octagons are for W56A and filled squares are for Sgr A*. The solid lines connecting the points have a slope of -2 , as expected for strong scattering. The dotted line is a prediction of the Taylor \& Cordes (1993) scattering model towards the Galactic Center.

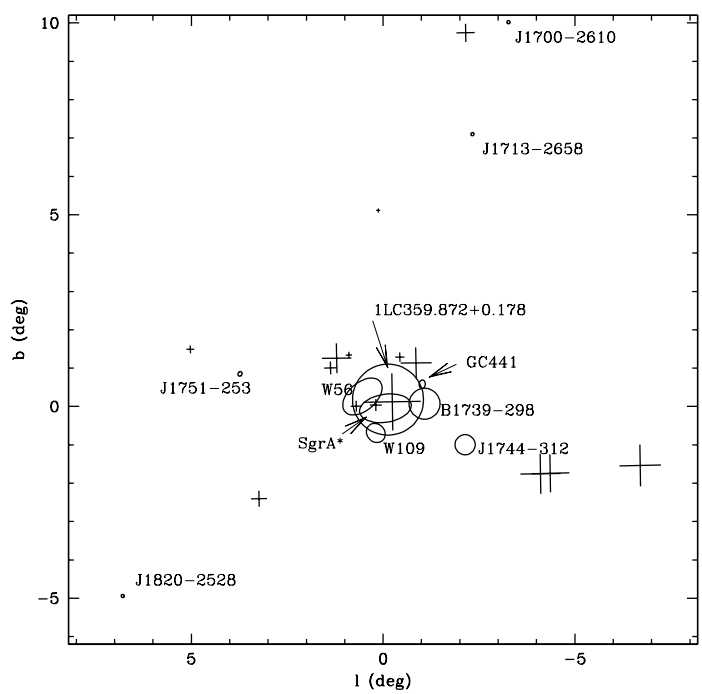

Fig. 3.- Scattered sources in the Galactic Center. The size of the ellipse or crosshair indicates the size of the scattered image at $1.6 \mathrm{GHz}$ with a scaling factor of $3^{\circ} \operatorname{arcsec}^{-1}$. The program sources are shown with their elliptical structure. Crosshairs indicate $\mathrm{OH} 1612 \mathrm{MHz}$ masers from van Langevelde et al. (1992). Labeled extragalactic sources are from references in the text. 
Table 1

Source Positions (J2000)

\begin{tabular}{ccc}
\hline \hline Source & RA & DEC \\
\hline GC441 & $17: 40: 54.5249$ & $-29: 29: 50.290$ \\
W56 & $17: 45: 52.4949$ & $-28: 20: 26.270$ \\
W109 & $17: 48: 45.6841$ & $-29: 07: 39.374$ \\
Sgr A* & $17: 45: 40.0385$ & $-29: 00: 28.104$ \\
\hline
\end{tabular}


TABle 2

Gaussian Components From VLBA Imaging

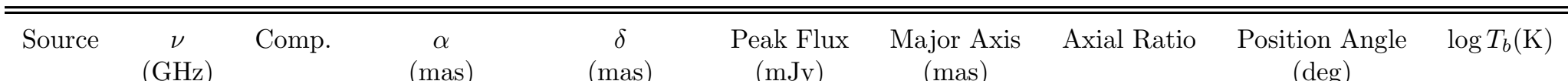

\begin{tabular}{|c|c|c|c|c|c|c|c|c|c|}
\hline \multirow[t]{2}{*}{ Sgr A* } & 5.0 & $\mathrm{~A}$ & $\ldots$ & $\ldots$ & $551.8 \pm 5.8$ & $47.2 \pm 1.0$ & $0.54 \pm 0.01$ & $81.2 \pm 1.1$ & 8.4 \\
\hline & 8.4 & $\mathrm{~A}$ & $\ldots$ & $\ldots$ & $766.4 \pm 2.5$ & $17.6 \pm 0.1$ & $0.51 \pm 0.01$ & $83.4 \pm 0.7$ & 9.5 \\
\hline \multirow[t]{5}{*}{ GC 441} & 2.3 & $\mathrm{~A}$ & $\ldots$ & $\ldots$ & $67.7 \pm 4.6$ & $37.6 \pm 13.3$ & $0.67 \pm 0.21$ & $-17.8 \pm 32.6$ & 7.6 \\
\hline & 5.0 & $\mathrm{~A}$ & $\ldots$ & $\ldots$ & $30.9 \pm 0.9$ & $7.7 \pm 1.5$ & $0.72 \pm 0.16$ & $4.0 \pm 11.1$ & 8.6 \\
\hline & 5.0 & $\mathrm{~B}$ & $-22.0 \pm 0.4$ & $31.5 \pm 0.7$ & $10.3 \pm 0.9$ & $8.2 \pm 1.2$ & & $\ldots$ & .. \\
\hline & 8.4 & $\mathrm{~A}$ & $\ldots$ & $\ldots$ & $21.6 \pm 0.9$ & $4.1 \pm 1.6$ & $0.54 \pm 0.23$ & $11.2 \pm 12.6$ & 9.1 \\
\hline & 8.4 & $\mathrm{~B}$ & $-22.5 \pm 0.8$ & $34.1 \pm 1.5$ & $5.5 \pm 0.9$ & $9.1 \pm 2.6$ & & $\ldots$ & .. \\
\hline \multirow[t]{3}{*}{ W56 } & 5.0 & $\mathrm{~A}$ & $\ldots$ & $\ldots$ & $88.8 \pm 3.1$ & $42.0 \pm 3.0$ & $0.60 \pm 0.04$ & $48.8 \pm 4.6$ & 7.7 \\
\hline & 5.0 & $\mathrm{~B}$ & $-100.7 \pm 4.4$ & $-207.3 \pm 7.1$ & $2.4 \pm 0.9$ & $\ldots$ & & $\ldots$ & . . \\
\hline & 8.4 & $\mathrm{~A}$ & $\ldots$ & $\ldots$ & $108.4 \pm 1.6$ & $15.4 \pm 0.5$ & $0.55 \pm 0.02$ & $50.8 \pm 3.9$ & 8.7 \\
\hline \multirow[t]{2}{*}{ W109 } & 5.0 & $\mathrm{~A}$ & $\ldots$ & $\ldots$ & $78.8 \pm 1.7$ & $18.3 \pm 0.9$ & $0.87 \pm 0.06$ & $138.8 \pm 24.9$ & 8.2 \\
\hline & 8.4 & $\mathrm{~A}$ & $\ldots$ & $\ldots$ & $60.2 \pm 0.9$ & $6.6 \pm 0.3$ & $0.78 \pm 0.03$ & $157.9 \pm 9.1$ & 9.0 \\
\hline
\end{tabular}


TABLE 3

Polarized and Total Flux from Vla Observations

\begin{tabular}{lrrrrrrr}
\hline \hline Source & $\begin{array}{c}I_{4.8} \\
(\mathrm{mJy})\end{array}$ & $\begin{array}{c}P_{4.8} \\
(\mathrm{mJy})\end{array}$ & $\begin{array}{c}\chi_{4.8} \\
(\mathrm{deg})\end{array}$ & $\begin{array}{c}I_{8.4} \\
(\mathrm{mJy})\end{array}$ & $\begin{array}{c}P_{8.4} \\
(\mathrm{mJy})\end{array}$ & $\begin{array}{c}\chi_{8.4} \\
(\mathrm{deg})\end{array}$ & $\begin{array}{c}\mathrm{RM} \\
\left(\mathrm{rad} \mathrm{m}^{-2}\right)\end{array}$ \\
\hline GC 441 & 44 & $<0.09$ & $\ldots$ & 27 & $<0.5$ & $\ldots$ & $\ldots$ \\
W56 & 104 & 2.2 & 79 & 136 & 4.0 & 47 & -217 \\
W109 & 98 & 0.59 & -26 & 95 & 1.1 & 28 & 376 \\
J1751-253 & 480 & 8.4 & -49 & 276 & 9.2 & -62 & -92 \\
\hline
\end{tabular}

TABLE 4

Differential Positions Between 5.0 and 8.4 GHz

\begin{tabular}{lrrrrrr}
\hline \hline & \multicolumn{4}{c}{ Centroid-Peak } & \multicolumn{3}{c}{ Differences } \\
\cline { 2 - 7 } Source & $\begin{array}{c}\Delta \alpha_{C} \\
(\text { mas })\end{array}$ & $\begin{array}{c}\Delta \delta_{C} \\
(\text { mas })\end{array}$ & $\begin{array}{c}\Delta \alpha_{X} \\
(\text { mas })\end{array}$ & $\begin{array}{c}\Delta \delta_{X} \\
(\text { mas })\end{array}$ & $\begin{array}{c}\Delta \alpha_{C X} \\
(\text { mas })\end{array}$ & $\begin{array}{c}\Delta \delta_{C X} \\
(\text { mas })\end{array}$ \\
\hline Sgr A & -0.1 & 0.0 & -0.1 & 0.2 & 0.1 & -0.2 \\
GC 441 & -4.0 & 6.3 & -0.9 & 1.2 & -3.2 & 5.1 \\
W56 & -4.0 & -7.5 & -0.2 & 0.2 & -3.8 & -7.7 \\
W109 & -0.1 & 0.3 & -0.0 & 0.0 & -0.1 & 0.4 \\
\hline
\end{tabular}

